\title{
Imaging practice in low-grade gliomas among European specialized centers and proposal for a minimum core of imaging
}

\author{
Christian F. Freyschlag ${ }^{1}$ - Sandro M. Krieg ${ }^{2}$ Johannes Kerschbaumer ${ }^{1} \cdot$ Daniel Pinggera $^{1} \cdot$ Marie-Therese Forster $^{3}$. \\ Dominik Cordier $^{4} \cdot$ Marco Rossi $^{5} \cdot$ Gabriele Miceli $^{6}$ - Alexandre Roux ${ }^{33,34}$. Andrés Reyes ${ }^{7,8,9} \cdot$ Silvio Sarubbo $^{10}$. \\ Anja Smits ${ }^{11,12}$. Joanna Sierpowska ${ }^{13,14}$. Pierre A. Robe ${ }^{15}$. Geert-Jan Rutten ${ }^{16}$. Thomas Santarius ${ }^{17}$. \\ Tomasz Matys $^{18}$ - Marc Zanello ${ }^{33,34}$. Fabien Almairac ${ }^{19} \cdot$ Lydiane Mondot $^{20}$ - Asgeir S. Jakola ${ }^{21,22} \cdot$ Maria Zetterling $^{23}$. \\ Adrià Rofes ${ }^{24,25}$. Gord von Campe ${ }^{26} \cdot$ Remy Guillevin $^{27}$. Daniele Bagatto ${ }^{28} \cdot$ Vincent Lubrano $^{29,30}$ - Marion Rapp ${ }^{31}$. \\ John Goodden ${ }^{40}$. Philip C. De Witt Hamer ${ }^{32}$. Johan Pallud ${ }^{33,34}$. Lorenzo Bello . Claudius Thomé $^{5}$ \\ Hugues Duffau ${ }^{35,36} \cdot$ Emmanuel Mandonnet ${ }^{37,38,39}$
}

Received: 5 February 2018 / Accepted: 29 May 2018 / Published online: 10 July 2018

(c) The Author(s) 2018

\begin{abstract}
Objective Imaging studies in diffuse low-grade gliomas (DLGG) vary across centers. In order to establish a minimal core of imaging necessary for further investigations and clinical trials in the field of DLGG, we aimed to establish the status quo within specialized European centers.

Methods An online survey composed of 46 items was sent out to members of the European Low-Grade Glioma Network, the European Association of Neurosurgical Societies, the German Society of Neurosurgery and the Austrian Society of Neurosurgery.

Results A total of 128 fully completed surveys were received and analyzed. Most centers $(n=96,75 \%)$ were academic and half of the centers $(n=64,50 \%)$ adhered to a dedicated treatment program for DLGG. There were national differences regarding the sequences enclosed in MRI imaging and use of PET, however most included T1 (without and with contrast, 100\%), T2 $(100 \%)$ and TIRM or FLAIR $(20,98 \%)$. DWI is performed by $80 \%$ of centers and $61 \%$ of centers regularly performed PWI. Conclusion A minimal core of imaging composed of T1 (w/wo contrast), T2, TIRM/FLAIR, PWI and DWI could be identified. All morphologic images should be obtained in a slice thickness of $\leq 3 \mathrm{~mm}$. No common standard could be obtained regarding advanced MRI protocols and PET.

Importance of the study We believe that our study makes a significant contribution to the literature because we were able to determine similarities in numerous aspects of LGG imaging. Using the proposed "minimal core of imaging" in clinical routine will facilitate future cooperative studies.
\end{abstract}

Keywords Low-grade glioma Imaging in LGG $\cdot$ Minimal core of imaging $\cdot$ Response criteria

\section{Introduction}

Despite published standards and guidelines on treatment and follow-up of diffuse low-grade glioma (DLGG) patients, daily practice frequently demonstrates the inconsistency of

Electronic supplementary material The online version of this article (https://doi.org/10.1007/s11060-018-2916-3) contains supplementary material, which is available to authorized users.

Christian F. Freyschlag

christian.freyschlag@i-med.ac.at

Extended author information available on the last page of the article imaging studies among centers [1,2]. As the volume of DLGG publications increases, the usefulness of singlecenter studies will become more limited, as these may be difficult to replicate. It has been argued that evidence-based practice in the field of DLGG cannot be derived from the standard methodology of oncological randomized clinical trials [3, 4]. Considering the low prevalence of DLGG [5] and the long survival of patients [6], sufficient data might be better collected by networks of centers working together collaboratively. Numerous demographic parameters, oncomolecular features and imaging data (including imaging DLGG growth rates of follow-up MRIs) will be required. 
Rigorous evaluation of care is additionally needed, for example to prove that maximum safe resection is not only key to oncological outcome, but also to establish and maintain a best possible quality of life [7,8]. The expectation that randomized oncological studies could add knowledge on these two questions is vanishing [3]. We are convinced that databases dedicated to DLGG research are required, which could include both retro- and prospective data.

The European Low-Grade Glioma Network (ELGGN) gathers surgical and neuroscience specialists from centers with dedicated teams treating DLGG patients. The network was founded 11 years ago to establish the link between all subspecialties involved in the field: neurosurgeons, neurooncologists, radiation therapists, neuropathologists, oncomolecular biologists, neuroradiologists, anaesthesiologists, speech therapists, neuropsychologists, and neuroscientists involved in functional brain mapping. Several collaborative studies have been previously published [3, 9-11].

The ELGGN is a powerful platform to address major issues in the management of DLGG. A survey [3] has been created in preparation of the 2015 Annual Meeting, which met the goal to identify points of consensus in patient management. Thus, the network should allow to highlight relevant questions for future studies and establish landmark projects in the interdisciplinary treatment of DLGG.

Following the initial survey, we now aimed to establish a comprehensive imaging survey, in order to investigate the consistency of DLGG imaging in specialized centers across Europe and to identify a "minimal core of imaging" to facilitate cooperative imaging projects within the network.

\section{Methods}

An online survey was created by a group of experts in imaging and treatment of DLGG. The use of published imaging guidelines $[1,12,13]$ was emphasized and local availability and usage of advanced imaging modalities was added to the survey. The survey was formatted on Survey Grid (EvaSys, Electric Paper Evaluationssysteme GmbH Lüneburg, Germany) and sent to all members of the ELGGN, the European Association of Neurosurgical Societies (EANS) plus the German (DGNC) and Austrian Society of Neurosurgery (ÖGNC). All recipients were members of the respective societies and it was specified that the questionnaire should be filled out by a multidisciplinary team. Participants were not asked to detail how any disagreements were adjudicated, precluding analysis of response heterogeneity at the center level. The survey contained 45 items (see Supplement 1), divided in descriptive data (i.e. amount of DLGG treated per year), radiological details (i.e. MRI sequences that are routinely performed) and questions regarding advanced imaging techniques (i.e. diffusion weighted imaging (DWI)).
In order to distinguish between dynamic susceptibility contrast perfusion imaging (DSC, named PWI in our survey) and dynamic contrast-enhanced MR perfusion (DCE, named Perfusion in our survey), two perfusion modalities were included in the survey, however, the question was answered inappropriately, showing that the nomenclature used for this particular question was misleading. Technical data on MRI scanner manufacturers, acquisition and recovery times, magnetic field strength and contrast agent dosage were not acquired. Further, the survey did not evaluate scientific justification of imaging protocols, it depicted a common denominator across many centers.

Further sections of the survey inquired the routine use of non-invasive mapping (i.e. resting state functional MRI (rs-fMRI) or navigated transcranial magnetic stimulation (nTMS)) and follow-up imaging protocols. The survey consisted of 28 single-choice, 7 multiple-choice and 10 items for free text answers.

In total, 148 data sets were received for detailed analysis. Data from outside Europe's geographical extension $(n=14)$ and incomplete surveys $(n=6)$ were excluded. Overall, 128 fully completed surveys were analyzed descriptively for this study. For additional information and geographical details of the respondents see Table 1.

\section{Results}

\section{Basic information}

The majority of responding teams worked in an academic hospital $(n=96,75 \%), 19 \%(n=25)$ were based in community hospitals, and $6 \%(\mathrm{n}=8)$ were located in a private hospital environment. Half of the centers $(64,50 \%)$ adhered to a dedicated MRI protocol for DLGG, while the others $(64,50 \%)$ did not. There was a broad range in the reported operative activity for each center (2-100 cases/year, average 25). The majority of centers treated less than 40 DLGG per year $(n=108,85 \%)$, whereas only 17 centers $(13 \%)$ reported to treat more than 40 . Three centers $(2 \%)$ can be considered very high volume centers with 100 DLGG per year (see Fig. 1). A total of 3032 DLGG are managed annually by the responding centers, the majority $(1714,62 \%)$ of which are treated in centers with dedicated DLGG programs compared to 1068 (38\%) in centers without. Of the centers treating 40 or more DLGG per year $65 \%$ used a dedicated DLGG protocol.

\section{Physicians involved in treatment of DLGG}

The survey showed that there is a variation in the composition of the multidisciplinary teams involved in DLGG management. The majority of centers (84\%) discussed their 
Table 1 Distribution of centers per country and average number of treated DLGG

\begin{tabular}{|c|c|c|c|c|}
\hline & $\begin{array}{l}\text { Number of centers } \\
\text { responded }\end{array}$ & $\%$ & $\begin{array}{l}\text { Avg. no of LGG per } \\
\text { center and year }\end{array}$ & Range \\
\hline \multicolumn{5}{|l|}{ Country of practice } \\
\hline Germany & 40 & 31 & 20 & $5-50$ \\
\hline Italy & 14 & 11 & 10 & $5-90$ \\
\hline France & 9 & 6 & 25 & $3-100$ \\
\hline Switzerland & 8 & 7 & 20 & $5-100$ \\
\hline United Kingdom & 8 & 6 & 30 & $10-60$ \\
\hline Austria & 7 & 5 & 20 & $6-40$ \\
\hline Spain & 7 & 5 & 5 & $3-20$ \\
\hline Netherlands & 6 & 5 & 25 & $15-40$ \\
\hline Portugal & 4 & 3 & 20 & $2-20$ \\
\hline Belgium & 3 & 2 & 15 & $15-30$ \\
\hline Greece & 3 & 2 & 25 & $10-30$ \\
\hline Poland & 3 & 2 & 25 & $20-30$ \\
\hline Czech Republic & 2 & 2 & 15 & 15 \\
\hline Russian Federation & 2 & 2 & 55 & $50-60$ \\
\hline Serbia & 2 & 2 & 15 & $10-20$ \\
\hline Sweden & 2 & 2 & 20 & 20 \\
\hline Bulgaria & 1 & $<1$ & 10 & 10 \\
\hline Denmark & 1 & $<1$ & 20 & 20 \\
\hline Hungary & 1 & $<1$ & $\mathrm{n} / \mathrm{a}$ & $\mathrm{n} / \mathrm{a}$ \\
\hline Lithuania & 1 & $<1$ & 30 & 30 \\
\hline Norway & 1 & $<1$ & 30 & 30 \\
\hline Romania & 1 & $<1$ & 10 & 10 \\
\hline Turkey & 1 & $<1$ & 20 & 20 \\
\hline Ukraine & 1 & $<1$ & 5 & 5 \\
\hline Use of 3T imaging & 128 & & & \\
\hline Always & 29 & 22.8 & & \\
\hline If available & 59 & 46.5 & & \\
\hline Only $1.5 \mathrm{~T}$ & 39 & 30.7 & & \\
\hline \multicolumn{5}{|l|}{ Identical MR scanner } \\
\hline Yes & 25 & 20.0 & & \\
\hline No & 24 & 19.2 & & \\
\hline Mostly yes & 72 & 57.6 & & \\
\hline \multirow[t]{2}{*}{ Mostly no } & 4 & 3.2 & & \\
\hline & & & \multicolumn{2}{|c|}{ Total number of patients } \\
\hline \multicolumn{5}{|c|}{ Slice thickness of T1 imaging (mm) } \\
\hline$<1.5$ & 66 & 53.7 & - & 1585 \\
\hline $1.6-3$ & 44 & 35.8 & - & 781 \\
\hline$>3$ & 13 & 10.6 & - & 356 \\
\hline \multicolumn{5}{|c|}{ Slice thickness of $\mathrm{T} 2$ imaging $(\mathrm{mm})$} \\
\hline$<1.5$ & 40 & 32.8 & - & 1027 \\
\hline $1.6-3$ & 54 & 44.3 & - & 1133 \\
\hline$>3$ & 28 & 23.0 & - & 542 \\
\hline \multicolumn{5}{|c|}{ Imaging intervals in relation to the amount of residual disease } \\
\hline \multicolumn{5}{|c|}{ No remnant (weeks) } \\
\hline$<12$ & 3 & 2.4 & & \\
\hline $12-24$ & 87 & 68.5 & & \\
\hline$>24$ & 15 & 11.8 & & \\
\hline 52 & 4 & 3.1 & & \\
\hline
\end{tabular}


Table 1 (continued)

\begin{tabular}{lll}
\hline & & \\
\hline$<10$ ml remnant (weeks) & & Total number of patients \\
$<12$ & 4 & 3.1 \\
$12-24$ & 91 & 71.7 \\
$>24$ & 12 & 9.4 \\
52 & 0 & 0.0 \\
$11-15$ ml remnant (weeks) & & \\
$<12$ & 4 & 3.1 \\
$12-24$ & 95 & 74.8 \\
$>24$ & 9 & 7.1 \\
52 & 0 & 0.0 \\
$>15$ ml remnant (weeks) & & \\
$<12$ & 7 & 5.5 \\
$12-24$ & 97 & 76.4 \\
$>24$ & 4 & 3.1 \\
52 & 0 & 0.0 \\
Unresectable LGG (weeks) & & \\
$<12$ & 1 & 0.8 \\
$12-24$ & 97 & 76.4 \\
$>24$ & 6 & 4.7 \\
52 & 3 & 2.4 \\
\hline
\end{tabular}

Use of imaging infrastructure and average slice thickness. Imaging intervals with respect to the amount of residual disease

Fig. 1 Distribution of annual DLGG throughout participating centers. Most centers treat less than 40 DLGG per year

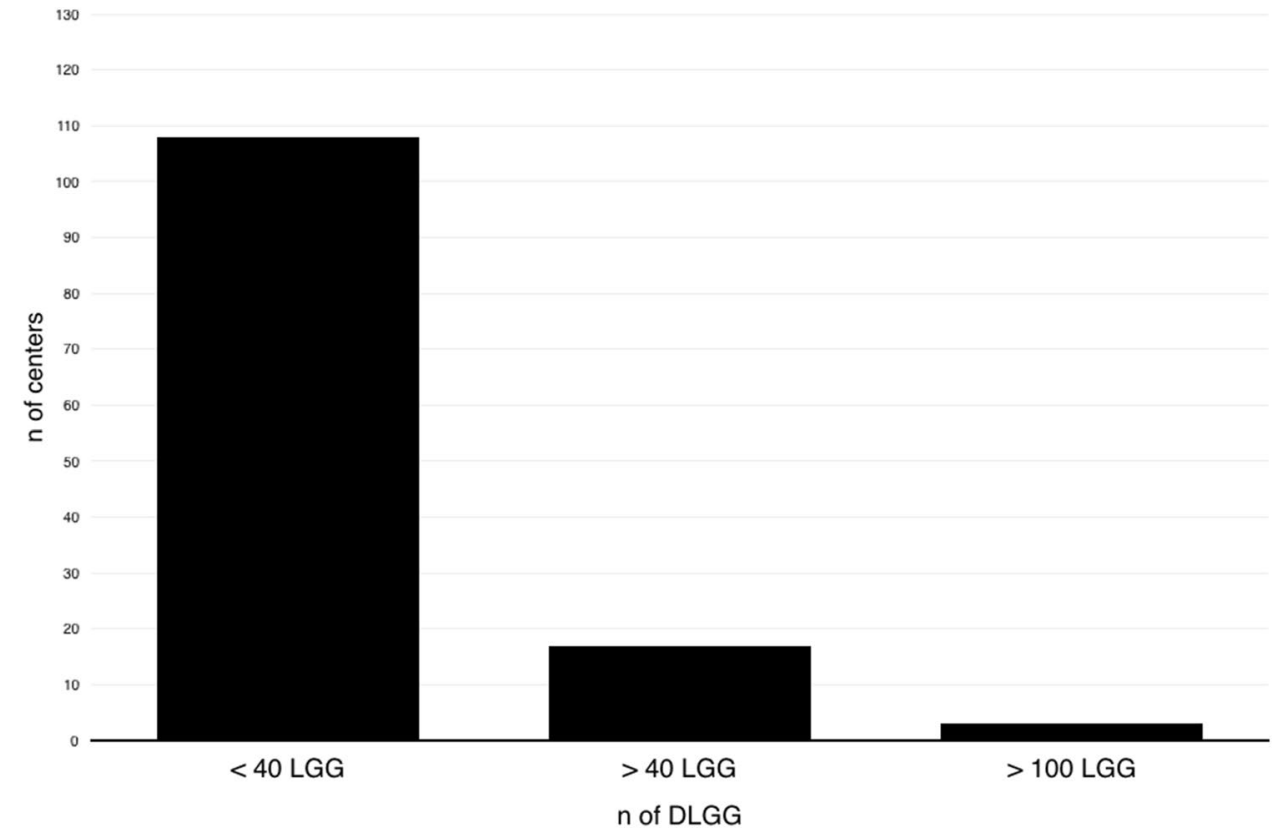

patients either before $(59,47 \%)$ or after surgical treatment $(47,37 \%)$. Interestingly, 20 centers (16\%) refrained from presenting every surgically treated DLGG in a multi-disciplinary tumor board, and 16 of these only discussed them if adjuvant treatment was advocated. Multi-disciplinary tumor boards consisted of several specialties: neurosurgeons were present in 99\%, followed by neuroradiologists (90\%), and radiation oncologists $(87 \%)$. Medical oncologists participated in $80 \%$ of neuro-oncological tumor boards, an additional $64 \%$ included a specialized neuro-oncologist. Nuclear medicine specialists, however, were available at the tumor board in only $32 \%$ of centers. 


\section{Imaging}

More than half (52\%) of the centers routinely used any recent MR imaging for treatment decisions and surgical treatment, without performing an MRI according to their own dedicated protocol. The imaging had to be carried out in a specialized neuroradiology unit (i.e. in a university hospital) in $17 \%$ of centers and $31 \%$ of centers always scanned their own dedicated protocol. The particular sequences applied by the center are summarized in Table 2. T1 imaging without and with Gadolinium contrast (T1 wo/w) and $\mathrm{T} 2$ weighted imaging was obtained in every center $(100 \%)$, whereas TIRM (turbo inversion resonance magnitude) and FLAIR (fluid attenuation inversion recovery) are performed in 20 and $98 \%$, respectively. Centers used a slice thickness $<1.5 \mathrm{~mm}$ in $52 \%$ of $\mathrm{T} 1$ and $32 \%$ of $\mathrm{T} 2$ images and a slice thickness of $\leq 3 \mathrm{~mm}$ in $89 \%$ of $\mathrm{T} 1$ and $78 \%$ of $\mathrm{T} 2$ images. Most of the centers (89\%) obtained DWI in every patient with an additional $80 \%$ obtaining apparent diffusion coefficient (ADC) maps routinely. Almost two-thirds of the respondents (61\%) applied perfusion weighted imaging (PWI) in daily routine.

Follow-up imaging of DLGG always included volumetric analysis (segmentation and approximation) in 45 centers (35\%) and linear measurement ( 3 axes on MRI) in 58 centers (45\%), and 26 centers (20\%) evaluated the deformation of present lesions or changes in shape to identify progression or regression. All measurements were performed by neuroradiologists in $59 \%$ opposed to $24 \%$ by neurosurgeons and $3 \%$ by neurooncologists. In $14 \%$ of centers, all members of the team performed measurements.

For interpretation of the response in DLGG, the RANO criteria [13] were used "always" in $15 \%$ of centers and "most of the time" in $46 \%$ of centers. $17 \%$ used the published criteria "hardly ever" and $22 \%$ refused to utilize them.

Table 2 Availability of MR-sequences in $\%$

\begin{tabular}{|c|c|c|c|c|c|c|c|c|c|c|c|c|c|c|c|c|c|c|c|}
\hline & $\begin{array}{c}\text { \# of } \\
\text { centers }\end{array}$ & $\begin{array}{c}\text { \# of } \\
\mathrm{LGG} / \mathrm{y}\end{array}$ & $\mathrm{T} 1+/-\mathrm{Gd}$ & $\mathrm{T} 2$ & TIRM & FLAIR & 3D-FLAIR & $\mathrm{T} 2^{*}$ & swı & PWI & DWI & $A D C$ & Perfusion & sv-1H-MRS & mv-1H-MRS & fMRI & rs-fMRI & PET & $\begin{array}{l}F / U \text { PET if } \\
\text { inital neg. }\end{array}$ \\
\hline Germany & 40 & 830 & 100 & 100 & 33 & 98 & 23 & 60 & 50 & 65 & 95 & 63 & 38 & 13 & 10 & 23 & 0 & 50 & 22 \\
\hline Italy & 14 & 273 & 100 & 100 & 7 & 93 & 71 & 71 & 21 & 64 & 100 & 79 & 71 & 36 & 29 & 36 & 7 & 62 & 14 \\
\hline France & 8 & 243 & 100 & 100 & 13 & 88 & 63 & 75 & 50 & 88 & 100 & 100 & 88 & 75 & 38 & 25 & 25 & 0 & 0 \\
\hline Switzerland & 8 & 230 & 100 & 100 & 13 & 88 & 13 & 25 & 88 & 38 & 100 & 75 & 50 & 25 & 0 & 25 & 0 & 50 & 25 \\
\hline United Kingdom & 8 & 280 & 100 & 100 & 0 & 88 & 25 & 38 & 13 & 50 & 100 & 88 & 88 & 25 & 25 & 13 & 0 & 14 & 0 \\
\hline Austria & 7 & 151 & 100 & 100 & 57 & 100 & 14 & 43 & 57 & 71 & 100 & 86 & 43 & 14 & 43 & 29 & 14 & 100 & 50 \\
\hline Spain & 7 & 58 & 100 & 100 & 14 & 100 & 71 & 71 & 43 & 86 & 86 & 71 & 86 & 57 & 14 & 29 & 14 & 17 & 17 \\
\hline Netherlands & 6 & 140 & 100 & 100 & 17 & 67 & 67 & 50 & 33 & 50 & 83 & 67 & 17 & 17 & 17 & 17 & 17 & 0 & 0 \\
\hline Portugal & 4 & 42 & 100 & 100 & 25 & 100 & 25 & 75 & 25 & 75 & 75 & 75 & 50 & 0 & 75 & 25 & 0 & 25 & 0 \\
\hline Belgium & 3 & 60 & 100 & 100 & 33 & 100 & 67 & 67 & 33 & 100 & 100 & 100 & 100 & 33 & 0 & 33 & 0 & 100 & 0 \\
\hline Greece & 3 & 65 & 100 & 100 & 0 & 100 & 67 & 33 & 100 & 67 & 100 & 67 & 67 & 0 & 67 & 33 & 33 & 0 & 0 \\
\hline Poland & 3 & 75 & 100 & 100 & 0 & 100 & 33 & 67 & 67 & 67 & 100 & 100 & 100 & 67 & 67 & 100 & 67 & 33 & 33 \\
\hline Czech Republic & 2 & 30 & 100 & 100 & 0 & 100 & 50 & 50 & 0 & 0 & 100 & 100 & 50 & 50 & 0 & 50 & 0 & 50 & 0 \\
\hline Russian Federation & 2 & 110 & 100 & 100 & 0 & 100 & 0 & 0 & 50 & 0 & 100 & 50 & 0 & 0 & 0 & 50 & 0 & 50 & 0 \\
\hline Serbia & 2 & 30 & 100 & 100 & 50 & 100 & 50 & 0 & 100 & 100 & 100 & 50 & 100 & 100 & 100 & 0 & 0 & 0 & 0 \\
\hline Sweden & 2 & 40 & 100 & 100 & 0 & 100 & 100 & 50 & 50 & 50 & 100 & 50 & 50 & 50 & 0 & 50 & 0 & 50 & 50 \\
\hline Bulgaria & 1 & 10 & 100 & 100 & 100 & 100 & 100 & 100 & 100 & 0 & 100 & 100 & 0 & 0 & 100 & 0 & 0 & 100 & 0 \\
\hline Denmark & 1 & 20 & 100 & 100 & 0 & 100 & 0 & 100 & 100 & 100 & 0 & 100 & 0 & 0 & 0 & 0 & 0 & 100 & 0 \\
\hline Hungary & 1 & $n / a$ & 100 & 100 & 0 & 100 & 100 & 100 & 100 & 100 & 100 & 100 & 100 & 0 & 100 & 100 & 0 & 0 & 0 \\
\hline Lithuania & 1 & 30 & 100 & 100 & 0 & 100 & 0 & 0 & 100 & 100 & 100 & 100 & 100 & 0 & 0 & 0 & 0 & 0 & 0 \\
\hline Norway & 1 & 30 & 100 & 100 & 0 & 100 & 100 & 100 & 100 & 100 & 100 & 100 & 100 & 100 & 100 & 0 & 0 & 0 & 0 \\
\hline Romania & 1 & 10 & 100 & 100 & 100 & 100 & 0 & 0 & 0 & 0 & 100 & 100 & 0 & 100 & 0 & 0 & 0 & 0 & 0 \\
\hline Turkey & 1 & 20 & 100 & 100 & 0 & 100 & 100 & 100 & 100 & 100 & 100 & 100 & 0 & 0 & 0 & 100 & 0 & 0 & 0 \\
\hline \multirow[t]{2}{*}{ Ukraine } & 1 & 5 & 100 & 100 & 0 & 100 & 0 & 0 & 0 & 0 & 0 & 0 & 0 & 0 & 0 & 0 & 0 & 0 & 0 \\
\hline & & & $\mathrm{T} 1+/-\mathrm{Gd}$ & $\mathrm{T} 2$ & TIRM & FLAIR & 3D-FLAIR & $\mathrm{T} 2^{*}$ & swı & PWI & DWI & $A D C$ & Perfusion & sv-1H-MRS & mv-1H-MRS & fMRI & rs-fMRI & PET & $\begin{array}{l}F / U \text { PET if } \\
\text { inital neg. }\end{array}$ \\
\hline Total & & 2782 & 2782 & 2782 & 538 & 2610 & 1043 & 1472 & 1350 & 1718 & 2673 & 2115 & 1548 & 769 & 617 & 765 & 205 & 1115 & 408 \\
\hline Total pre/postop & & 5564 & 5564 & 5564 & 1076 & 5220 & 2086 & 2944 & 2700 & 3436 & 5346 & 4231 & 3096 & 1538 & 1234 & 1530 & 410 & & \\
\hline Mean percentage & & & 100 & 100 & 19 & 97 & 47 & 53 & 58 & 61 & 89 & 80 & 54 & 32 & 33 & 31 & 7 & 33 & 9 \\
\hline
\end{tabular}

$T 1+/-G d$ T1 weighted imaging with and without Gadolinium contrast, TIRM turbo inversion resonance magnitude, FLAIR fluid attenuated inversion recovery, $3 D$-FLAIR multiplanar reconstruction of FLAIR, $T 2 *$ gradient-echo T2 with susceptibility, $S W I$ susceptibility weighted imaging, $P W I$ perfusion weighted imaging, $D W I$ diffusion weighted imaging, $A D C$ automated diffusion coefficient, $s v-1 H$ - $M R S$ single-voxel Proton magnetic resonance spectroscopy, $m v-1 H$-MRS multi-voxel Proton magnetic resonance spectroscopy, $f M R I$ functional MRI, $r s-f M R I$ restingstate functional MRI, PET positron emission tomography 
Although $81 \%$ of centers specified to adjust their imaging intervals according to the tumor's previous growth rate, more than $75 \%$ of centers perform follow-up imaging in intervals of 12-24 weeks (see Table 1) in all cases presented, regardless of the amount of residual tumor.

\section{Advanced imaging}

Additional advanced sequences, like MR spectroscopy (MRS) were handled variably throughout the centers. Onethird of centers routinely obtained data from single-voxel spectroscopy, another third applied a multi-voxel spectroscopy in their MRI protocol.

The question "Do you perform amino acid PET in suspected low-grade glioma?" was answered positively in 33\% of all respondents. However, centers tend to discard PET imaging in case of initially negative PET scans. Under these circumstances only $9 \%$ would repeat PET imaging later. Interestingly, some countries like Austria and Belgium had a $100 \%$ rate of initial PET imaging, whereas French centers performed no PET. Assessment of treatment response and progression was mostly (80\%) done with MRI (T2/FLAIR for response, $\mathrm{T} 1 \mathrm{wo} / \mathrm{w}$ for progression), whereas $14 \%$ relied on the combination of MRI and amino-acid PET (aaPET; if initially positive). Six percent of centers performed MRI and aaPET regardless of initial PET presentation. The survey investigated which imaging the centers would rely on to decide whether the tumor has undergone anaplastic transformation. Unsurprisingly, there is no consensus on the imaging modalities used for detection of anaplastic transformation. Most centers used combinations of either T1wo/w with MRS and PWI or T1wo/w with FET-PET (see Table 3).

The number of MRI studies that would be available for further investigation was calculated for every MR sequence based on the number of patients treated annually in all responding centers. Up to 2800 studies should be available for analysis every year (see Table 2 ).

\section{Functional MRI and non-invasive brain mapping}

The survey demonstrated a wide heterogeneity regarding the use of functional MRI (fMRI) for patients with DLGG.

Thirty-one percent routinely used fMRI for every patient, while resting-state fMRI is acquired in only $7 \%$. Half of the centers $(50 \%)$ used fMRI for both, clinical and research purposes, $42 \%$ exclusively clinical, and $6 \%$ only for research. A minority of $2 \%$ used fMRI for didactic purposes in training of students or residents.

The final part of the survey evaluated brain mapping and the respective technique of choice: fMRI, nTMS, or intraoperative direct electrical cortical and subcortical stimulation. 102 centers $(80 \%)$ preferred invasive intraoperative direct electrical cortical and subcortical stimulation over
Table 3 Choice of imaging modalities for detection of malignant transformation

\begin{tabular}{lcr}
\hline & $\mathrm{n}$ & $\%$ \\
\hline T1 + PWI + MRS & 23 & 18 \\
T1 + FET-PET & 20 & 16 \\
T1 + PWI & 20 & 16 \\
T1 & 19 & 15 \\
T1 + PWI + FET-PET & 10 & 8 \\
T1 + PWI + MRS + FET-PET & 8 & 6 \\
T1 + MRS & 4 & 3 \\
T1 + MRS + FET-PET & 3 & 2 \\
T1 + PWI + MRS + other & 3 & 2 \\
FET-PET & 3 & 2 \\
PWI+ MRS & 3 & 2 \\
T1 + PWI+ other & 2 & 2 \\
T1 + other & 2 & 2 \\
other & 8 & 6 \\
Other & & \\
Biopsy/resection & 7 & 6 \\
Evaluate changes in growth rate/volumet- & 3 & 1 \\
$\quad$ ric expansion & & 1 \\
F-DOPA PET & 1 & \\
Arterial-spin labelling MRI & 1 & \\
DWI/ADC & 1 & \\
\hline
\end{tabular}

noninvasive procedures. 8 centers $(6 \%)$, however, would have chosen nTMS, whereas 18 centers (14\%) preferred fMRI. The number of centers using magnetoencephalography (MEG) was low, which fits the distribution of the technique. $87 \%$ of centers don't use MEG or do not own one, although $71 \%$ recognized the scientific possibilities of MEG or thought it would be nice to have.

\section{Discussion}

Our survey revealed a high level of homogeneity in DLGG imaging workup throughout Europe. Nonetheless, we were able to identify heterogeneities that need to be highlighted. Of note, questions were not designed as detailed individual cases and we acknowledge that this method might have provoked heterogeneity of responses.

\section{Minimal core of imaging}

Ellingson et al. [1] proposed a standardized brain imaging protocol for tumor patients. We support the principle of creating standardized protocols for DLGG patients. This should include minimum imaging datasets, recommended imaging frequency and recommendations about additional sequences. The goal of establishing a minimal core of imaging would 
be to allow further investigations with uniform imaging throughout different countries. This would enable to include numerous patients in future prospective cohorts, ensuring a sufficient volume of data to perform big data analysis [14].

The minimal core of imaging in DLGG needs to take regional and national differences regarding technical standards and reimbursement into account. Therefore, the following imaging algorithm is proposed: MR imaging should incorporate sequences for morphologic descriptive analysis and those focusing on potential malignant transformation. $\mathrm{T} 1 \mathrm{wo} / \mathrm{w}$ and $\mathrm{T} 2$ images were used by all centers and therefore represent the cornerstone of morphologic imaging. In addition, TIRM or FLAIR sequences should be obtained and can also be used for volumetric assessment of DLGG. Although several recommendations set the minimum level of slice thickness in T2 and TIRM/FLAIR imaging to $\leq 4 \mathrm{~mm}$ [1], it is known that slice thickness is key for accurate volumetric and treatment response assessment [15-17]. Perfusion weighted imaging (PWI) is used frequently for assessment of treatment response and detection of anaplastic transformation $[18,19]$. In our survey, response and transformation were predominantly determined with MRI. T1 (with contrast) and PWI were used in 87 and 55\%, respectively. Diffusion weighted imaging (DWI), and especially the computed ADC has been used to investigate malignant transformation [20] and is applied in up to $90 \%$ of centers.

We therefore recommend that the minimal MRI sequence dataset should consist of T1wo/w, T2 and TIRM/FLAIR, all in low slice thickness $(\leq 3 \mathrm{~mm})$ to facilitate volumetric assessment and further include PWI and DWI to predict malignant transformation. Based on this survey, we cannot make any recommendation regarding the use of additional advanced imaging techniques (spectroscopy, fMRI, PET).

\section{Imaging intervals}

More than 75\% adhered to follow-up imaging intervals of 12 to 24 weeks and $81 \%$ adjusted their imaging intervals depending on the initial growth rate of the tumor [21], which is in accordance with current guidelines [2]. It has been shown that the velocity of tumor expansion is a strong predictor of the patient's prognosis [21]. However, no studies have addressed the question of how often MRI should be obtained during follow-up, especially with regard to the heterogeneity of DLGG. Although early postoperative FLAIR is known to overestimate the volume of residual tumor [22], most authors recommend an early postoperative MRI within $72 \mathrm{~h}$ [23-25] to determine the extent of resection and visualize possible postoperative complications. Follow-up imaging intervals of 12-24 weeks are recommended with longer intervals for cases of "less aggressive" tumours [1, 13, 26]. The definition of "aggressiveness", however, varies in the literature.

\section{Standardized response assessment protocols}

Only $15 \%$ of the centers reported to use RANO criteria for low-grade gliomas [13] thoroughly in all their DLGG cases for the interpretation of treatment response, while $46 \%$ of centers do so "most of the time". Thus, $39 \%$ of centers do not routinely apply these criteria. RANO criteria include T1wo/w, T2/FLAIR, development of new lesions, clinical status, and steroid use in order to categorize treatment effects in complete response, partial response, stable disease, and progressive disease. Since DLGG constitutes a slowly progressive disease, these criteria were defined in order to achieve a standardized common ground for the definitions of treatment responses and endpoints in clinical trials. Measurement of tumor diameter has various shortcomings, not only the high intra- and interobserver variability [27], but also the known problem of head positioning during acquisition of the MRI $[16,17]$. Then, assuming that many centers are performing volumetric assessment of tumors, how should treatment response be defined? Translating volume to diameter $\left(\mathrm{D}=(2 \times \mathrm{V})^{\wedge}(1 / 3)\right)$ is a fundamental step. In contrast to the assessment of tumor diameters, the concept of volume-derived mean diameter overcomes the above mentioned confounding factors. Moreover, the curve showing evolution of mean diameter with time can be easily analyzed by applying a linear fit. Following the curve of the diameter as a function of time is a more sensitive way to monitor treatment response than applying RANO criteria [21, 28, 29]. Indeed, the major concern about RANO criteria is that pretreatment dynamics is not integrated in the definition of the different response categories. However, it seems obvious that putting down the tumor growth rate to $0 \mathrm{~mm}$ /year with chemotherapy, while its pretreatment value was $6 \mathrm{~mm} /$ year, should be interpreted as a response, whereas RANO criteria would interpret this as "stable disease".

In contrast to this highly standardized protocol, our survey focused more on the clinical routine in an attempt to accurately reflect every day practice in the treatment of DLGG across Europe. Although the highly standardized approach provided by the RANO criteria is not systematically applied, our data demonstrate that at least the imaging studies, comply in most centers with the RANO-defined protocols.

Additionally, other factors should also be interpreted into our decision making, such as cognitive testing, psychological burden of disease, and seizure activity.

\section{Malignant transformation}

There is no consensus regarding the radiological malignant transformation in DLGG throughout Europe. Newly apparent contrast enhancement indicating breakdown of the blood brain barrier represents the classical sign of 
malignant transformation (MT) of these tumors [30], but preceding changes in advanced MRI investigations may allow identification of patients at risk up to 12 months earlier [31]. Simple measurement of growth rate [19, 32, 33] and integration of MR spetroscopy [18, 19, 34] are used routinely in most of the centers as predictors of tumor transformation. Both, proton- and phosphorus spectroscopy, available in numerous centers, have been proven to correlate with Ki67 and IDH1 mutation [35]. Perfusion measurements, and in particular the determination of relative cerebral blood volume (rCBV), seems to correlate with the vascularity determined at histopathological examination [31]. Arising of lactate resonance is predictive of the increase of $\mathrm{rCBV}$ up to 1.75 , this parameter is predictive for a dramatic decrease of overall survival [36-38], and are indicators of MT that may be identified months before apparent contrast enhancement. Likewise, ADC can be used for discrimination of tumor subtypes and raising suspicion of malignization [20]. Nevertheless, both techniques are only supported by a low level of evidence [20].

\section{Positron emission tomography (PET)}

The use of amino acid PET (aaPET) was surprisingly variable between centers and countries. While Austria and Belgium performed PET in 100\% of cases, none of the Dutch or French centers used PET at all. In contrast to patients with WHO grades III/IV gliomas, the evidence for aaPET to monitor patients with WHO grade II gliomas is limited [39, 40]. Most WHO grade II gliomas are nonenhancing with infiltrating tumor borders, and so several studies demonstrated the usefulness of aaPET in defining tumor extent or malignant transformation [41-45]. This has been demonstrated and validated in series for $11 \mathrm{C}$ MET, 18F-FET, and 18F-FDOPA PET [46]. Although MRI is the standard of care in following DLGG patients, its reliability in distinguishing tumor tissue from treatment effects is limited [47]. Transient blood-brain barrier alteration with contrast enhancement after radiotherapy with or without concomitant Temozolomide, for example, can mimic tumor progression. There are numerous reasons for the restricted application of PET: (1) While 18FFDG is used routinely, access to aaPET is limited [46]. (2) Another obstacle to withhold patients and healthcare professionals from PET is limited reimbursement. In our survey, $9 \%$ of centers repeated aaPET scans during follow-up, even if they were initially negative. However, there are several groups advocating to perform aaPET in all cases of DLGG [48], notwithstanding the initial uptake behavior.

\section{fMRI and noninvasive neurophysiological imaging}

fMRI has repeatedly been shown to harbor a low specificity and sensitivity for any presurgical evaluation [49-53]. Since the tumor itself impairs oxygenation levels in its surrounding fMRI is not a reliable surrogate marker for neuronal activity in DLGG patients [50, 51, 54]. Nevertheless, eloquence is thoroughly based on fMRI in as much as $16 \%$ of centers.

Yet, $80 \%$ of European centers prefer invasive intraoperative direct electrical cortical and subcortical stimulation over noninvasive procedures for the determination of eloquent cortex, which reflects commonly agreed practice and level of evidence [55-57].

With the still small but increasing distribution of nTMS, some centers chose this technique for surgical decision making, which also illustrates the highly specialized nature of the enrolled centers. Although nTMS is a noninvasive modality, several reports proved not only the accuracy but also the feasibility of using it as a highly reliable tool for presurgical planning and intraoperative navigation $[23,58,59]$ of primary motor functions. While non-invasive mapping can be reliably performed for primary motor functions (taskbased fMRI, nTMS), this is currently not true for higher order functions (movement coordination, language, spatial consciousness, mentalizing, ...) [60].

MEG was also rarely used. Nonetheless, despite its limited availability and high costs, its usefulness for presurgical planning and follow-up has repeatedly been reported [61-63].

Irrespective of the used modality, noninvasive evaluation of eloquent function, especially if adjacent to or within the tumor is mandatory in order to identify the optimal time point for re-resection. This is even more relevant in the contrast of tumor-induced cortical reorganization potentially allowing gross total resection of previously unresectable tumors [64-68].

In the near future, ongoing developments might be capable to achieve a change in imaging practice. Alongside with huge developments of machine learning using conventional MRI, which suppose the robustness of standardized sequences; an increased interest of metabolic multinuclear MR imaging and integration of multiparametric data into realistic metabolic-dynamic mathematical models is noticed.

\section{Strengths and limitations}

The survey solely focused on imaging modalities for preoperative workup and follow up investigations of DLGG patients. The large number of centers involved and the high conformity of surgical treatment within participants of the ELGGN represent the major strength of this study. Up to 2800 MRIs per year would be available in this network. 
Online surveys are limited by the accuracy of the given statements and their representation of a multidisciplinary team cannot be guaranteed in online survey, which limits the reliability of the results. Questions were not designed as detailed individual cases and we acknowledge that this method might have provoked heterogeneity of responses. Additionally, the study did not aim to investigate the outcome of DLGG treatment.

\section{Conclusion}

It appears mandatory to standardize the initial management and follow-up of DLGG in order to maximize the number of included patients in future multicentric studies. If a certain proximity of imaging protocol throughout Europe is already present, this work emphasizes the need to clarify important questions such as assessment of treatment response or detection of DLGG malignant transformation. ELGGN can help to resolve important issues and to promote a better care for patients suffering from DLGG.

Acknowledgements Open access funding provided by University of Innsbruck and Medical University of Innsbruck.

\section{Compliance with ethical standards}

Conflict of interest On behalf of all authors we declare no conflict of interest.

Open Access This article is distributed under the terms of the Creative Commons Attribution 4.0 International License (http://creativeco mmons.org/licenses/by/4.0/), which permits unrestricted use, distribution, and reproduction in any medium, provided you give appropriate credit to the original author(s) and the source, provide a link to the Creative Commons license, and indicate if changes were made.

\section{References}

1. Ellingson BM, Bendszus M, Boxerman J, Barboriak D, Erickson BJ, Smits M, Nelson SJ, Gerstner E, Alexander B, Goldmacher G, Wick W, Vogelbaum M, Weller M, Galanis E, Kalpathy-Cramer J, Shankar L, Jacobs P, Pope WB, Yang D, Chung C, Knopp MV, Cha S, van den Bent MJ, Chang S, Yung WK, Cloughesy TF, Wen PY, Gilbert MR, Jumpstarting Brain Tumor Drug Development Coalition Imaging Standardization Steering C (2015) Consensus recommendations for a standardized Brain Tumor Imaging Protocol in clinical trials. Neuro Oncol 17:1188-1198. https://doi. org/10.1093/neuonc/nov095

2. Weller M, van den Bent M, Tonn JC, Stupp R, Preusser M, Cohen-Jonathan-Moyal E, Henriksson R, Le Rhun E, Balana C, Chinot O, Bendszus M, Reijneveld JC, Dhermain F, French P, Marosi C, Watts C, Oberg I, Pilkington G, Baumert BG, Taphoorn MJB, Hegi M, Westphal M, Reifenberger G, Soffietti R, Wick W, European Association for Neuro-Oncology Task Force on G (2017) European Association for Neuro-Oncology (EANO) guideline on the diagnosis and treatment of adult astrocytic and oligodendroglial gliomas. Lancet Oncol 18: e315-e329 https://doi. org/10.1016/S1470-2045(17)30194-8

3. Mandonnet E, Wager M, Almairac F, Baron M-H, Blonski M, Freyschlag CF, Barone F, Fontaine D, Pallud J, Hegi M, Viegas C, Zetterling M, Spena G, Goodden J, Rutten G-J, Taillandier L, Foroglu N, Darlix A, Skrap M, Martino J, von Campe G, Madadaki C, Gayat E, de Witt Hamer P, Gil Robles S, Sarubbo S, Santorius T, Bello L, Forster M-T, Duffau H (2017) Survey on current practice within the European Low-Grade Glioma Network: where do we stand and what is the next step? Neuro-Oncol Pract. https://doi.org/10.1093/nop/npw031

4. Duffau H, Mandonnet E, Pallud J, Krieg SM, Gil Robles S, De Witt Hamer P, Schucht P, Zetterling M, Santarius T, Colle H, Ryan M, Smits A, von Campe G, Bello L, Forster MT, Sarubbo S, Spena G, Baron MH, Yordanova YN, Darlix A, Viegas C, Almairac F, Martino J, Goodden J, Chumas P, Freyschlag CF, Robe P, Wager M, Polivka M, Giakoumettis D, Robert E, Guillevin R, Grivas A, Fontaine D, van Greemen K, Lubrano V, Rutten GJ, Barone F, Rofes A, Rech F, Rigau V, Wagemakers M, Papagno C, Aerts A, Visch-Brink E, Tate M, Garg N, Klein M, Satoer D, De Witte E, van Brussel L, Reyes A, van Ierschot F, Veenstra W, Snijders T, Taillandier L, Blonski M (2017) Evidenced-based medicine in glioma: molecular biology is only part of the story. Lancet Oncol. https://doi.org/10.1016/ s1470-2045(17)30510-7

5. Wohrer A, Waldhor T, Heinzl H, Hackl M, Feichtinger J, GruberMosenbacher U, Kiefer A, Maier H, Motz R, Reiner-Concin A, Richling B, Idriceanu C, Scarpatetti M, Sedivy R, Bankl HC, Stiglbauer W, Preusser M, Rossler K, Hainfellner JA (2009) The Austrian Brain Tumour Registry: a cooperative way to establish a population-based brain tumour registry. J Neurooncol 95:401411. https://doi.org/10.1007/s11060-009-9938-9

6. Duffau H, Taillandier L (2015) New concepts in the management of diffuse low-grade glioma: proposal of a multistage and individualized therapeutic approach. Neuro Oncol 17:332-342. https ://doi.org/10.1093/neuonc/nou153

7. Jakola AS, Unsgard G, Myrmel KS, Kloster R, Torp SH, Lindal S, Solheim O (2012) Low grade gliomas in eloquent locationsimplications for surgical strategy, survival and long term quality of life. PLoS ONE 7:e51450. https://doi.org/10.1371/journ al.pone.0051450

8. Moritz-Gasser S, Herbet G, Maldonado IL, Duffau H (2012) Lexical access speed is significantly correlated with the return to professional activities after awake surgery for low-grade gliomas. J Neurooncol 107:633-641. https://doi.org/10.1007/s1106 0-011-0789-9

9. Beez T, Boge K, Wager M, Whittle I, Fontaine D, Spena G, Braun S, Szelenyi A, Bello L, Duffau H, Sabel M, European Low Grade Glioma N (2013) Tolerance of awake surgery for glioma: a prospective European Low Grade Glioma Network multicenter study. Acta Neurochir (Wien) 155:1301-1308. https://doi.org/10.1007/ s00701-013-1759-0

10. Szelenyi A, Bello L, Duffau H, Fava E, Feigl GC, Galanda M, Neuloh G, Signorelli F, Sala F Workgroup for Intraoperative Management in Low-Grade Glioma Surgery within the European Low-Grade Glioma (2010) Intraoperative electrical stimulation in awake craniotomy: methodological aspects of current practice. Neurosurg Focus 28:E7. https://doi.org/10.3171/2009.12.FOCUS 09237

11. De Witt Hamer PC, Hendriks EJ, Mandonnet E, Barkhof F, Zwinderman AH, Duffau H (2013) Resection probability maps for quality assessment of glioma surgery without brain location bias. PLoS ONE 8:e73353. https://doi.org/10.1371/journal.pone.00733 53

12. Wen PY, Macdonald DR, Reardon DA, Cloughesy TF, Sorensen AG, Galanis E, Degroot J, Wick W, Gilbert MR, Lassman AB, 
Tsien C, Mikkelsen T, Wong ET, Chamberlain MC, Stupp R, Lamborn KR, Vogelbaum MA, van den Bent MJ, Chang SM (2010) Updated response assessment criteria for high-grade gliomas: response assessment in neuro-oncology working group. J Clin Oncol 28:1963-1972. https://doi.org/10.1200/JCO.2009.26.3541

13. van den Bent MJ, Wefel JS, Schiff D, Taphoorn MJB, Jaeckle K, Junck L, Armstrong T, Choucair A, Waldman AD, Gorlia T, Chamberlain M, Baumert BG, Vogelbaum MA, Macdonald DR, Reardon DA, Wen PY, Chang SM, Jacobs AH (2011) Response assessment in neuro-oncology (a report of the RANO group): assessment of outcome in trials of diffuse low-grade gliomas. Lancet Oncol 12:583-593. https://doi.org/10.1016/ s1470-2045(11)70057-2

14. Ostrom QT, Bauchet L, Davis FG, Deltour I, Fisher JL, Langer CE, Pekmezci M, Schwartzbaum JA, Turner MC, Walsh KM, Wrensch MR, Barnholtz-Sloan JS (2014) The epidemiology of glioma in adults: a "state of the science" review. Neuro Oncol 16:896-913. https://doi.org/10.1093/neuonc/nou087

15. Bauknecht HC, Romano VC, Rogalla P, Klingebiel R, Wolf C, Bornemann L, Hamm B, Hein PA (2010) Intra- and interobserver variability of linear and volumetric measurements of brain metastases using contrast-enhanced magnetic resonance imaging. Invest Radiol 45:49-56. https://doi.org/10.1097/ RLI.0b013e3181c02ed5

16. Reuter M, Gerstner ER, Rapalino O, Batchelor TT, Rosen B, Fischl B (2014) Impact of MRI head placement on glioma response assessment. J Neurooncol 118:123-129. https://doi.org/10.1007/ s11060-014-1403-8

17. Schmitt P, Mandonnet E, Perdreau A, Angelini ED (2013) Effects of slice thickness and head rotation when measuring glioma sizes on MRI: in support of volume segmentation versus two largest diameters methods. J Neurooncol 112:165-172. https://doi. org/10.1007/s11060-013-1051-4

18. Bobek-Billewicz B, Stasik-Pres G, Hebda A, Majchrzak K, Kaspera W, Jurkowski M (2014) Anaplastic transformation of low-grade gliomas (WHO II) on magnetic resonance imaging. Folia Neuropathol 52:128-140

19. Hlaihel C, Guilloton L, Guyotat J, Streichenberger N, Honnorat J, Cotton F (2010) Predictive value of multimodality MRI using conventional, perfusion, and spectroscopy MR in anaplastic transformation of low-grade oligodendrogliomas. J Neurooncol 97:73-80. https://doi.org/10.1007/s11060-009-9991-4

20. Fouke SJ, Benzinger T, Gibson D, Ryken TC, Kalkanis SN, Olson JJ (2015) The role of imaging in the management of adults with diffuse low grade glioma: a systematic review and evidence-based clinical practice guideline. J Neurooncol 125:457-479. https://doi. org/10.1007/s11060-015-1908-9

21. Pallud J, Blonski M, Mandonnet E, Audureau E, Fontaine D, Sanai N, Bauchet L, Peruzzi P, Frenay M, Colin P, Guillevin R, Bernier V, Baron MH, Guyotat J, Duffau H, Taillandier L, Capelle L (2013) Velocity of tumor spontaneous expansion predicts long-term outcomes for diffuse low-grade gliomas. Neuro Oncol 15:595-606. https://doi.org/10.1093/neuonc/nos331

22. Belhawi SM, Hoefnagels FW, Baaijen JC, Aliaga ES, Reijneveld JC, Heimans JJ, Barkhof F, Vandertop WP, Hamer PC (2011) Early postoperative MRI overestimates residual tumour after resection of gliomas with no or minimal enhancement. Eur Radiol 21:1526-1534. https://doi.org/10.1007/s00330-011-2081-y

23. Takahashi S, Jussen D, Vajkoczy P, Picht T (2012) Plastic relocation of motor cortex in a patient with LGG (low grade glioma) confirmed by NBS (navigated brain stimulation). Acta Neurochir (Wien) 154:2003-2008. https://doi.org/10.1007/s00701-0121492-0 discussion 2008

24. Bette S, Kaesmacher J, Huber T, Delbridge C, Ringel F, BoeckhBehrens T, Meyer B, Zimmer C, Kirschke JS, Gempt J (2016) Value of early postoperative FLAIR volume dynamic in glioma with no or minimal enhancement. World Neurosurg 91:548-559. e541. https://doi.org/10.1016/j.wneu.2016.03.034

25. Albert FK, Forsting M, Sartor K, Adams HP, Kunze S (1994) Early postoperative magnetic resonance imaging after resection of malignant glioma: objective evaluation of residual tumor and its influence on regrowth and prognosis. Neurosurgery $34: 45-60$; discussion $60-41$

26. Chang EF, Clark A, Jensen RL, Bernstein M, Guha A, Carrabba G, Mukhopadhyay D, Kim W, Liau LM, Chang SM, Smith JS, Berger MS, McDermott MW (2009) Multiinstitutional validation of the University of California at San Francisco low-grade glioma prognostic scoring system. J Neurosurg 111:203-210. https://doi. org/10.3171/2009.2.JNS081101

27. Sorensen AG, Patel S, Harmath C, Bridges S, Synnott J, Sievers A, Yoon YH, Lee EJ, Yang MC, Lewis RF, Harris GJ, Lev M, Schaefer PW, Buchbinder BR, Barest G, Yamada K, Ponzo J, Kwon HY, Gemmete J, Farkas J, Tievsky AL, Ziegler RB, Salhus MR, Weisskoff R (2001) Comparison of diameter and perimeter methods for tumor volume calculation. J Clin Oncol 19:551-557. https://doi.org/10.1200/JCO.2001.19.2.551

28. Pallud J, Mandonnet E, Duffau H, Kujas M, Guillevin R, Galanaud D, Taillandier L, Capelle L (2006) Prognostic value of initial magnetic resonance imaging growth rates for World Health Organization grade II gliomas. Ann Neurol 60:380-383. https:// doi.org/10.1002/ana.20946

29. Izquierdo C, Alentorn A, Idbaih A, Simo M, Kaloshi G, Ricard D, Barritault M, Meyronet D, Bruna J, Honnorat J, Delattre JY, Ducray F (2017) Long-term impact of temozolomide on 1p/19qcodeleted low-grade glioma growth kinetics. J Neurooncol. https ://doi.org/10.1007/s11060-017-2677-4

30. Pallud J, Capelle L, Taillandier L, Fontaine D, Mandonnet E, Guillevin R, Bauchet L, Peruzzi P, Laigle-Donadey F, Kujas M, Guyotat J, Baron MH, Mokhtari K, Duffau H (2009) Prognostic significance of imaging contrast enhancement for WHO grade II gliomas. Neuro Oncol 11:176-182. https://doi.org/10.1215/15228 517-2008-066

31. Danchaivijitr N, Waldman AD, Tozer DJ, Benton CE, Brasil Caseiras G, Tofts PS, Rees JH, Jager HR (2008) Low-grade gliomas: do changes in rCBV measurements at longitudinal perfusionweighted MR imaging predict malignant transformation? Radiology 247:170-178. https://doi.org/10.1148/radiol.2471062089

32. Rees J, Watt H, Jager HR, Benton C, Tozer D, Tofts P, Waldman A (2009) Volumes and growth rates of untreated adult low-grade gliomas indicate risk of early malignant transformation. Eur $\mathbf{J}$ Radiol 72:54-64. https://doi.org/10.1016/j.ejrad.2008.06.013

33. Cochereau J, Herbet G, Rigau V, Duffau H (2016) Acute progression of untreated incidental WHO Grade II glioma to glioblastoma in an asymptomatic patient. J Neurosurg 124:141-145. https://doi. org/10.3171/2014.12.jns141851

34. Jalbert LE, Neill E, Phillips JJ, Lupo JM, Olson MP, Molinaro AM, Berger MS, Chang SM, Nelson SJ (2016) Magnetic resonance analysis of malignant transformation in recurrent glioma. Neuro Oncol 18:1169-1179. https://doi.org/10.1093/neuonc/ now008

35. Guillevin R, Menuel C, Duffau H, Kujas M, Capelle L, Aubert A, Taillibert S, Idbaih A, Pallud J, Demarco G, Costalat R, HoangXuan K, Chiras J, Vallee JN (2008) Proton magnetic resonance spectroscopy predicts proliferative activity in diffuse low-grade gliomas. J Neurooncol 87:181-187. https://doi.org/10.1007/s1106 0-007-9508-y

36. Shiroishi MS, Habibi M, Rajderkar D, Yurko C, Go JL, Lerner A, Mogensen MA, Kim PE, Boyko OB, Zee CS, Law M (2011) Perfusion and permeability MR imaging of gliomas. Technol Cancer Res Treat 10:59-71. https://doi.org/10.7785/tcrt.2012.500180

37. Essig M, Anzalone N, Combs SE, Dorfler A, Lee SK, Picozzi P, Rovira A, Weller M, Law M (2012) MR imaging of neoplastic 
central nervous system lesions: review and recommendations for current practice. AJNR Am J Neuroradiol 33:803-817. https://doi. org/10.3174/ajnr.A2640

38. Essig M, Nguyen TB, Shiroishi MS, Saake M, Provenzale JM, Enterline DS, Anzalone N, Dorfler A, Rovira A, Wintermark M, Law M (2013) Perfusion MRI: the five most frequently asked clinical questions. AJR Am J Roentgenol 201:W495-510. https:// doi.org/10.2214/ajr.12.9544

39. Voges J, Herholz K, Holzer T, Wurker M, Bauer B, Pietrzyk U, Treuer H, Schroder R, Sturm V, Heiss WD (1997) 11C-methionine and 18F-2-fluorodeoxyglucose positron emission tomography: a tool for diagnosis of cerebral glioma and monitoring after brachytherapy with 125I seeds. Stereotact Funct Neurosurg 69:129-135

40. Wyss M, Hofer S, Bruehlmeier M, Hefti M, Uhlmann C, Bartschi E, Buettner UW, Roelcke U (2009) Early metabolic responses in temozolomide treated low-grade glioma patients. J Neurooncol 95:87-93. https://doi.org/10.1007/s11060-009-9896-2

41. Dunet V, Rossier C, Buck A, Stupp R, Prior JO (2012) Performance of 18F-fluoro-ethyl-tyrosine (18F-FET) PET for the differential diagnosis of primary brain tumor: a systematic review and Metaanalysis. J Nucl Med 53:207-214. https://doi.org/10.2967/ jnumed.111.096859

42. Gempt J, Bette S, Ryang YM, Buchmann N, Peschke P, Pyka T, Wester HJ, Forster S, Meyer B, Ringel F (2015) 18F-fluoro-ethyltyrosine positron emission tomography for grading and estimation of prognosis in patients with intracranial gliomas. Eur J Radiol 84:955-962. https://doi.org/10.1016/j.ejrad.2015.01.022

43. Jansen NL, Suchorska B, Wenter V, Eigenbrod S, Schmid-Tannwald C, Zwergal A, Niyazi M, Drexler M, Bartenstein P, Schnell O, Tonn JC, Thon N, Kreth FW, la Fougere C (2014) Dynamic $18 \mathrm{~F}-\mathrm{FET}$ PET in newly diagnosed astrocytic low-grade glioma identifies high-risk patients. J Nucl Med 55:198-203. https://doi. org/10.2967/jnumed.113.122333

44. Jansen NL, Suchorska B, Wenter V, Schmid-Tannwald C, Todica A, Eigenbrod S, Niyazi M, Tonn JC, Bartenstein P, Kreth FW, la Fougere C (2015) Prognostic significance of dynamic 18F-FET PET in newly diagnosed astrocytic high-grade glioma. J Nucl Med 56:9-15. https://doi.org/10.2967/jnumed.114.144675

45. Villani V, Carapella CM, Chiaravalloti A, Terrenato I, Piludu F, Vidiri A, Schillaci O, Floris R, Marzi S, Fabi A, Pace A (2015) The Role of PET [18F]FDOPA in Evaluating Low-grade Glioma. Anticancer Res 35:5117-5122

46. Albert NL, Weller M, Suchorska B, Galldiks N, Soffietti R, Kim MM, la Fougere C, Pope W, Law I, Arbizu J, Chamberlain MC, Vogelbaum M, Ellingson BM, Tonn JC (2016) Response Assessment in Neuro-Oncology working group and European Association for Neuro-Oncology recommendations for the clinical use of PET imaging in gliomas. Neuro Oncol 18:1199-1208. https://doi. org/10.1093/neuonc/now058

47. Kumar AJ, Leeds NE, Fuller GN, Van Tassel P, Maor MH, Sawaya RE, Levin VA (2000) Malignant gliomas: MR imaging spectrum of radiation therapy- and chemotherapy-induced necrosis of the brain after treatment. Radiology 217:377-384. https://doi. org/10.1148/radiology.217.2.r00nv36377

48. Unterrainer M, Schweisthal F, Suchorska B, Wenter V, SchmidTannwald C, Fendler WP, Schuller U, Bartenstein P, Tonn JC, Albert NL (2016) Serial 18F-FET PET imaging of primarily 18F-FET-negative glioma: does it make sense? J Nucl Med 57:1177-1182. https://doi.org/10.2967/jnumed.115.171033

49. Bartos R, Jech R, Vymazal J, Petrovicky P, Vachata P, Hejcl A, Zolal A, Sames M (2009) Validity of primary motor area localization with fMRI versus electric cortical stimulation: a comparative study. Acta Neurochir (Wien) 151:1071-1080. https://doi. org/10.1007/s00701-009-0368-4

50. Giussani C, Roux FE, Ojemann J, Sganzerla EP, Pirillo D, Papagno C (2010) Is preoperative functional magnetic resonance imaging reliable for language areas mapping in brain tumor surgery? Review of language functional magnetic resonance imaging and direct cortical stimulation correlation studies. Neurosurgery 66:113-120. https://doi.org/10.1227/01.NEU.0000360392.15450 .C9

51. Kuchcinski G, Mellerio C, Pallud J, Dezamis E, Turc G, RigauxViode O, Malherbe C, Roca P, Leclerc X, Varlet P, Chretien F, Devaux B, Meder JF, Oppenheim C (2015) Three-tesla functional MR language mapping: comparison with direct cortical stimulation in gliomas. Neurology 84:560-568. https://doi.org/10.1212/ WNL.0000000000001226

52. Morrison MA, Tam F, Garavaglia MM, Hare GM, Cusimano MD, Schweizer TA, Das S, Graham SJ (2016) Sources of variation influencing concordance between functional MRI and direct cortical stimulation in brain tumor surgery. Front Neurosci 10:461. https://doi.org/10.3389/fnins.2016.00461

53. Stevens MT, Clarke DB, Stroink G, Beyea SD, D’Arcy RC (2016) Improving fMRI reliability in presurgical mapping for brain tumours. J Neurol Neurosurg Psychiatry 87:267-274. https://doi. org/10.1136/jnnp-2015-310307

54. Ille S, Sollmann N, Hauck T, Maurer S, Tanigawa N, Obermueller T, Negwer C, Droese D, Boeckh-Behrens T, Meyer B, Ringel F, Krieg SM (2015) Impairment of preoperative language mapping by lesion location: a functional magnetic resonance imaging, navigated transcranial magnetic stimulation, and direct cortical stimulation study. J Neurosurg 123:314-324. https://doi. org/10.3171/2014.10.JNS141582

55. Duffau H, Lopes M, Arthuis F, Bitar A, Sichez JP, Van Effenterre R, Capelle L (2005) Contribution of intraoperative electrical stimulations in surgery of low grade gliomas: a comparative study between two series without (1985-96) and with (1996-2003) functional mapping in the same institution. J Neurol Neurosurg Psychiatry 76:845-851. https://doi.org/10.1136/jnnp.2004.04852 0

56. De Witt Hamer PC, Robles SG, Zwinderman AH, Duffau H, Berger MS (2012) Impact of intraoperative stimulation brain mapping on glioma surgery outcome: a meta-analysis. J Clin Oncol 30:2559-2565. https://doi.org/10.1200/JCO.2011.38.4818

57. Rofes A, Mandonnet E, Godden J, Baron MH, Colle H, Darlix A, de Aguiar V, Duffau H, Herbet G, Klein M, Lubrano V, Martino J, Mathew R, Miceli G, Moritz-Gasser S, Pallud J, Papagno C, Rech F, Robert E, Rutten GJ, Santarius T, Satoer D, Sierpowska J, Smits A, Skrap M, Spena G, Visch E, De Witte E, Zetterling M, Wager M (2017) Survey on current cognitive practices within the European Low-Grade Glioma Network: towards a European assessment protocol. Acta Neurochir (Wien) 159:1167-1178. https://doi.org/10.1007/s00701-017-3192-2

58. Ille S, Sollmann N, Butenschoen VM, Meyer B, Ringel F, Krieg SM (2016) Resection of highly language-eloquent brain lesions based purely on rTMS language mapping without awake surgery. Acta Neurochir (Wien) 158:2265-2275. https://doi.org/10.1007/ s00701-016-2968-0

59. Kawashima A, Krieg SM, Faust K, Schneider H, Vajkoczy P, Picht $\mathrm{T}$ (2013) Plastic reshaping of cortical language areas evaluated by navigated transcranial magnetic stimulation in a surgical case of glioblastoma multiforme. Clin Neurol Neurosurg 115:2226-2229. https://doi.org/10.1016/j.clineuro.2013.07.012

60. Krieg SM, Lioumis P, Makela JP, Wilenius J, Karhu J, Hannula H, Savolainen P, Lucas CW, Seidel K, Laakso A, Islam M, Vaalto S, Lehtinen H, Vitikainen AM, Tarapore PE, Picht T (2017) Protocol for motor and language mapping by navigated TMS in patients and healthy volunteers; workshop report. Acta Neurochir (Wien) 159:1187-1195. https://doi.org/10.1007/s00701-017-3187-z

61. Tarapore PE, Findlay AM, Honma SM, Mizuiri D, Houde JF, Berger MS, Nagarajan SS (2013) Language mapping with navigated repetitive TMS: proof of technique and validation. 
Neuroimage 82:260-272. https://doi.org/10.1016/j.neuroimage 2013.05.018

62. Tarapore PE, Martino J, Guggisberg AG, Owen J, Honma SM, Findlay A, Berger MS, Kirsch HE, Nagarajan SS (2012) Magnetoencephalographic imaging of resting-state functional connectivity predicts postsurgical neurological outcome in brain gliomas. Neurosurgery 71:1012-1022. https://doi.org/10.1227/NEU.0b013 e31826d2b78

63. Tarapore PE, Tate MC, Findlay AM, Honma SM, Mizuiri D, Berger MS, Nagarajan SS (2012) Preoperative multimodal motor mapping: a comparison of magnetoencephalography imaging, navigated transcranial magnetic stimulation, and direct cortical stimulation. J Neurosurg 117:354-362. https://doi. org/10.3171/2012.5.JNS112124

64. Robles SG, Gatignol P, Lehericy S, Duffau H (2008) Long-term brain plasticity allowing a multistage surgical approach to World Health Organization Grade II gliomas in eloquent areas. J Neurosurg 109:615-624. https://doi.org/10.3171/JNS/2008/109/10/0615

65. Ius T, Angelini E, Thiebaut de Schotten M, Mandonnet E, Duffau H (2011) Evidence for potentials and limitations of brain plasticity using an atlas of functional resectability of WHO grade II gliomas: towards a "minimal common brain". Neuroimage 56:9921000. https://doi.org/10.1016/j.neuroimage.2011.03.022

66. Ille S, Kulchytska N, Sollmann N, Wittig R, Beurskens E, Butenschoen VM, Ringel F, Vajkoczy P, Meyer B, Picht T, Krieg SM (2016) Hemispheric language dominance measured by repetitive navigated transcranial magnetic stimulation and postoperative course of language function in brain tumor patients. Neuropsychologia 91:50-60. https://doi.org/10.1016/j.neuropsychologi a.2016.07.025

67. Southwell DG, Hervey-Jumper SL, Perry DW, Berger MS (2016) Intraoperative mapping during repeat awake craniotomy reveals the functional plasticity of adult cortex. J Neurosurg 124:1460 1469. https://doi.org/10.3171/2015.5.JNS142833

68. Conway N, Wildschuetz N, Moser T, Bulubas L, Sollmann N, Tanigawa N, Meyer B, Krieg SM (2017) Cortical plasticity of motor-eloquent areas measured by navigated transcranial magnetic stimulation in patients with glioma. J Neurosurg 127:981-991. https://doi.org/10.3171/2016.9.JNS161595

\section{Affiliations}

\section{Christian F. Freyschlag ${ }^{1}$ - Sandro M. Krieg ${ }^{2}$. Johannes Kerschbaumer ${ }^{1}$. Daniel Pinggera ${ }^{1} \cdot$ Marie-Therese Forster $^{3}$. Dominik Cordier $^{4}$. Marco Rossi ${ }^{5}$. Gabriele Miceli ${ }^{6}$. Alexandre Roux ${ }^{33,34}$. Andrés Reyes ${ }^{7,8,9}$. Silvio Sarubbo ${ }^{10}$. Anja Smits ${ }^{11,12}$. Joanna Sierpowska ${ }^{13,14}$. Pierre A. Robe ${ }^{15}$. Geert-Jan Rutten ${ }^{16}$. Thomas Santarius ${ }^{17}$. Tomasz Matys ${ }^{18} \cdot$ Marc Zanello $^{33,34} \cdot$ Fabien Almairac ${ }^{19} \cdot$ Lydiane Mondot $^{20} \cdot$ Asgeir S. Jakola $^{21,22} \cdot$ Maria Zetterling $^{23}$. Adrià Rofes ${ }^{24,25}$. Gord von Campe ${ }^{26} \cdot$ Remy Guillevin $^{27}$. Daniele Bagatto ${ }^{28}$. Vincent Lubrano ${ }^{29,30}$ - Marion Rapp ${ }^{31}$. John Goodden ${ }^{40}$. Philip C. De Witt Hamer ${ }^{32}$. Johan Pallud ${ }^{33,34}$. Lorenzo Bello . Claudius Thomé $^{5}$ Hugues Duffau ${ }^{35,36} \cdot$ Emmanuel Mandonnet $^{37,38,39}$}

1 Department of Neurosurgery, Medical University of Innsbruck, Anichstrasse 35, 6020 Innsbruck, Austria

2 Department of Neurosurgery, Klinikum rechts der Isar, Technische Universität München, Munich, Germany

3 Department of Neurosurgery, Goethe University Hospital, Frankfurt am Main, Germany

4 Department of Neurosurgery, Universitätsspital Basel, Basel, Switzerland

5 Neurosurgical Oncology Unit, Humanitas Research Hospital, IRCCS, Milan, Italy

6 Center for Mind/Brain Sciences, University of Trento, Rovereto, Italy

7 European Master's in Clinical Linguistics (EMCL), University of Groningen, Groningen, The Netherlands

8 EMCL University of Potsdam, Potsdam, Germany

9 Neuroscience Institute, and Laboratory of Experimental Psychology, Faculty of Psychology, El Bosque University, Bogotá, Colombia

10 Division of Neurosurgery, Structural and Functional Connectivity Lab Project, "S. Chiara" Hospital, APSS, Trento, Italy

11 Department of Clinical Neuroscience, Institute of Neuroscience and Physiology, Sahlgrenska Academy, University of Gothenburg, Gothenburg, Sweden
12 Department of Neuroscience, Neurology, Uppsala University, Uppsala, Sweden

13 Cognition and Brain Plasticity Unit, Bellvitge Biomedical Research Institute (IDIBELL), University of Barcelona, Barcelona, Spain

14 Department of Cognition, Development and Education Psychology, Barcelona, Spain

15 Department of Neurology and Neurosurgery, Rudolf Magnus Brain Institute, University Medical Center of Utrecht, Utrecht, The Netherlands

16 Department of Neurosurgery, Elisabeth-Tweesteden Hospital, Tilburg, The Netherlands

17 Department of Neurosurgery, Addenbrooke's Hospital, University of Cambridge, Cambridge, UK

18 Department of Radiology, Addenbrooke's Hospital, University of Cambridge, Cambridge, UK

19 Neurosurgery Department, Hôpital Pasteur 2, University Hospital of Nice, Nice, France

20 Radiology Department, Hôpital Pasteur 2, University Hospital of Nice, Nice, France

21 Department of Neurosurgery, Sahlgrenska University Hospital, Gothenburg, Sweden

22 Department of Clinical Neuroscience, Institute of Neuroscience and Physiology, Sahlgrenska Academy, Gothenburg, Sweden 
23 Department of Neurosurgery, Institution of Neuroscience, Uppsala University Hospital, Uppsala, Sweden

24 Global Brain Health Institute, Trinity College Dublin, Dublin, Ireland

Department of Cognitive Science, Johns Hopkins University, Baltimore, USA

26 Department of Neurosurgery, Medical University Graz, Graz, Austria

27 DACTIM, UMR CNRS 7348, Université de Poitiers et CHU de Poitiers, Poitiers, France

28 Neuroradiology Department, University Hospital Santa Maria della Misericordia, Udine, Italy

29 Department of Neurosurgery, CHU Toulouse, Toulouse, France

30 ToNIC, Toulouse NeuroImaging Center, Université de Toulouse, Inserm, UPS, Toulouse, France

31 Department of Neurosurgery, Medical Faculty, Heinrich Heine University, Düsseldorf, Germany
32 VU University Medical Center, Amsterdam, The Netherlands

33 Department of Neurosurgery, Sainte-Anne Hospital, Paris Descartes University, Sorbonne Paris Cité, Paris, France

34 Inserm U894, IMA-Brain, Centre de Psychiatrie et Neurosciences, Paris, France

35 Department of Neurosurgery, Hôpital Gui de Chauliac, Montpellier Medical University Center, Montpellier, France

36 Institute of Neuroscience of Montpellier, INSERM U1051, University of Montpellier, Montpellier, France

37 Department of Neurosurgery, Lariboisière Hospital, APHP, Paris, France

38 University Paris 7, Paris, France

39 IMNC, UMR 8165, Orsay, France

40 Department of Neurosurgery, The General Infirmary at Leeds, Leeds, West Yorkshire, UK 